\title{
Novel technology using polyacrylamide membranes for biological separations
}

\author{
Smitha Kenchath*, Benjamin Lim, Krithika Kumar, Wong Tee Wee \\ PRIME Biologics Pte Ltd, 41 Science Park Road, \#01-01C, The Gemini (Lobby B), Singapore 117610
}

*Corresponding author: smitha.kenchath@primebiologics.com

\section{Article history}

Received 5 January 2019

Revised 7 Mac 2019

Accepted 28 October 2019

Published Online 2 February 2020

\begin{abstract}
PRIME technology is a novel preparative electrophoretic isolation method for the purification of biological proteins based on molecular size and charge. Molecular charge, generated by the choice of a suitable buffered $\mathrm{pH}$ system, is employed in combination with polyacrylamide hydrogel membranes of selected porosities to separate molecules. By using both size and charge as restrictive factors for purification, the PRIME method is able to isolate molecules with high yield and purity. In this paper, we discuss the use of PRIME technology in the purification of plasma proteins. In the present work, albumin at $93 \%$ purity was purified at high yield $(98 \%)$. Using albumindepleted plasma for further processing of immunoglobulins (IVIG), we were able to obtain an IVIG product yield as high as $92 \%$ and purity as high as $88 \%$. Since PRIME does not use solvents or require sub-zero temperatures and is well-suited for plasma purification from modest plasma volumes, we propose the application of PRIME as a cost-effective and safe alternative to Cohn fractionation.
\end{abstract}

Keywords: Biological separations; electrophoretic isolation; polyacrylamide membrane; restriction membrane; separation membrane.

\section{INTRODUCTION}

Crucial plasma-derived therapeutic products have been in clinical use for several decades, with many new therapeutics being isolated from plasma. Human plasma is a key source of therapeutic proteins used to treat patients with life-threatening diseases or conditions, including burns, hemophilia, shock, trauma, neurological disorders, and innate or episodic immune deficiencies. Albumin, immunoglobulins (IgG or IVIG), and Factor VIII have shown the greatest commercial and medical significance [1]. Purification of these and other plasma proteins are commonly performed using Cohn fractionation, a method largely unchanged since its introduction in the 1940s, with only minor modifications made to improve the process [2].

Despite its status as the standard technology for plasma protein purification, Cohn has several disadvantages, particularly for its use in developing countries. A Cohn facility is expansive and expensive and is most cost-effective when there is a large and continuous volume of plasma supply. Additionally, Cohn is best suited for source plasma obtained via plasmapheresis, a process in which plasma is collected directly from donors, often times paid, using machines that extract whole blood, retain the plasma, and return red blood cells back to the donor. An alternative source of plasma is generated as a by-product of whole blood donations from which red blood cells and platelets have been removed [3]. In most developing countries, where plasma is obtained as recovered plasma, it is available in smaller quantities and therefore neither the plasma supply volume nor the resources justify the use of a Cohn facility.

Paradoxically, however, there is a growing increase in demand for plasma therapeutics in these countries. This results in reliance on either (a) foreign fractionation facilities, commonly referred to as toll fractionation, to process domestic plasma, resulting in increased product cost and product shortage or (b) purchase of finished plasma proteins on the global market. Most foreign fractionation facilities "pool" plasma from multiple sources to meet the volume required for Cohn fractionation, creating a concern for plasma safety. Additionally, pooled plasma is mainly suited only for the production of polyvalent $\operatorname{IgG}$ titers, and not for the enrichment of specific antibodies. Given these disadvantages and the rapidly growing demand for plasma proteins in emerging markets, an alternative method for low cost, high yield purification of both source and recovered plasma is urgently required.

PRIME is a polyacrylamide membrane-based, preparative electrophoresis technology platform developed to purify macromolecules from complex biological samples. PRIME technology combines size and charge-based separation to purify biomolecules. Size-based separation is achieved by using two types of membranes (separation membranes and restriction membranes) with specific molecular weight cut-off (MWCO) or pore sizes to separate a target molecule. Molecules with molecular weights less than the MWCO of the restriction and separation membranes will transfer across the membranes, while those with molecular weights greater than that of the MWCO will not. Charge-based separation occurs in conjunction with size-based separation and relies on the fact that a molecule's charge is dependent on its isoelectric point (pI) and the $\mathrm{pH}$ of the solution in which it is present. By using the appropriate buffer to confer a net positive, negative, or neutral charge on the target molecule, it is electrophoretically separated, with positively charged molecules migrating towards the negative electrode and negatively 
charged molecules migrating towards the positive electrode (Fig. 1). This system can be combined with traditional upstream and downstream processing to increase efficiency and purity of the process.

The key factors in successful purifying target molecules using PRIME technology are (i) analyse the starting material for its composition, including impurities, (ii) choose an appropriate buffer to confer varying charges to the target molecule and the impurities, and (iii) design membranes and cartridges to selectively isolate the target molecule. Target molecules can be purified under conditions where the protein is in a native or altered state. The PRIME system can be easily scaled for increased sample volume by increasing the membrane surface area, increasing the number of membranes in a disposable cartridge, or increasing the number of disposable cartridges in series to allow operation using a known volume of the starting material. The use of a disposable cartridge also prevents any crossprocess contamination and allows the remaining hardware to be easily decontaminated through a validated cleaning process.

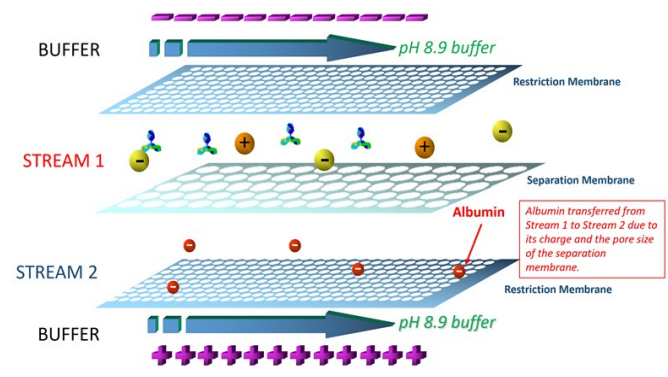

Fig. 1 Schematic outlines the principle behind PRIME purification of albumin from human plasma. A separation membrane is sandwiched between two restriction membranes. The membranes serve to isolate the target protein from other molecules based on specific MWCO. In addition, the restriction membranes isolate the buffer stream from the product stream. The buffers serve to confer a charge on the target molecules and impurities. Albumin, in this case, is conferred a negative charge and therefore, moves towards the positive electrode.

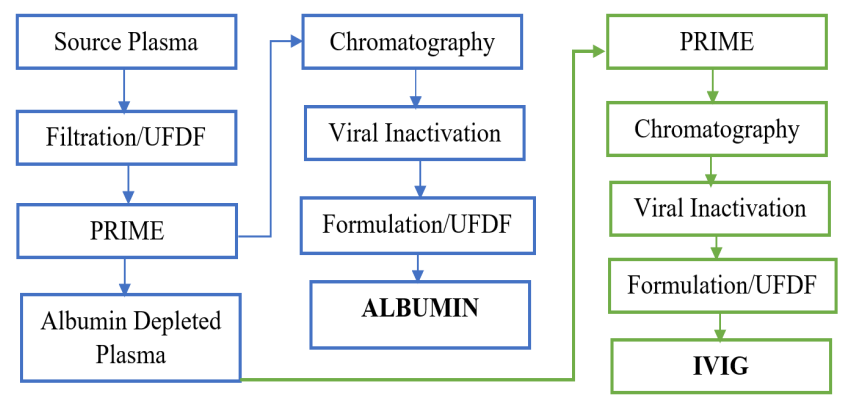

Fig. 2 Process albumin and IVIG purification from human plasma using PRIME technology. Blue boxes indicate the steps for the purification of albumin from source plasma, while green boxes indicate the steps for the purification of IVIG from albumin-depleted plasma.

The main advantage of a PRIME unit is that it can operate efficiently with an annual production volume of 50,000 litres, which falls within the range of plasma produced by many developing countries. Use of a domestic PRIME facility also reduces shipping, transport, and storage costs. Finally, domestic processing of plasma will allow for matching of donor and recipient populations, which may increase the therapeutic efficacy of some plasma therapeutics, particularly IgGs and hyperimmunes.

Based on the properties and versatility of PRIME, we propose that PRIME can be applied to replace Cohn fractionation as an efficient and cost-effective method for the production of plasma-derived therapeutics in developing countries, as well as developed countries with relatively small populations. In this study, we demonstrate a new method for the isolation of albumin and IVIG with yield and purity comparable or superior to conventional methods. We also discuss PRIME as an alternative to Cohn fractionation for the purification of other plasma proteins.

\section{MATERIALS AND METHODS}

Starting materials. The key starting materials are human plasma (FFP,ADMA, U.S.), boric acid $\left(\mathrm{H}_{3} \mathrm{BO}_{3}\right.$, Merck Millipore), human albumin reference standards, EP (European Pharmacopoeia), sodium hydroxide $(\mathrm{NaOH}$, Merck Millipore), sodium chloride ( $\mathrm{NaCl}$, Sigma Aldrich), human IgG reference standards, EP (European Pharmacopoeia), ethanol $\left(\mathrm{C}_{2} \mathrm{H}_{5} \mathrm{OH}\right.$, Merck Millipore), acrylamide (Sigma Aldrich), bisacrylamide (Sigma Aldrich), ammonium persulfate (APS, Sigma Aldrich), tetramethylethylenediamine (TEMED, Sigma Aldrich), and polyethylene terephthalate (PET, CAS 25038-59-9).

\section{Polyacrylamide Membrane}

Polyacrylamide membranes with defined pore sizes are manufactured by the reaction of acrylamide with a bifunctional cross-linking agent. This involves the free radical polymerization of acrylamide and bisacrylamide, with APS and TEMED acting as the free radical generator and catalyst, respectively. The membranes are formed on a substrate of PET fabric, separated by plastic sheets, which gives it a characteristic thickness and glossiness to the polymerized membranes. All solutions used in the polymerisation process are prepared with purified water under ambient condition. Acrylamide and bisacrylamide solutions have to be degassed before use, as the presence of oxygen will inhibit the polymerisation of acrylamide since oxygen traps free radicals.

The process of casting membranes involves pouring the acrylamide mixture after addition of initiator into the casting tank, before layering the different configurations of glass plates, plastic sheets and PET substrate in the tank. The solution is then left aside for polymerization and curing.

After polymerisation is completed, the membranes are cut into appropriate sizes and assembled into cartridges. A set/combination of restriction membranes and separation membranes is called a PRIME cartridge. Typically, these cartridges contain two restriction membranes and one separation membrane. Commercial-scale cartridges are produced for use in the ProTrans 50, a commercial scale PRIME processing unit. Prior to their use, membranes are visually inspected for holes and/or bubbles. Both cartridge leak tests and membrane transfer rate/retention rates are checked prior to the release of a cartridge.

\section{Filtration and UF/DF}

Fresh frozen plasma (FFP) was thawed in a $2-8{ }^{\circ} \mathrm{C}$ cold room for $48 \pm$ $6 \mathrm{hrs}$. FFP was then placed in a $37^{\circ} \mathrm{C}$ water bath for approximately 15 additional minutes to ensure that it was completely thawed. The thawed plasma was filtered through $5 \mu \mathrm{m}$ filters to remove any coarse precipitates. In the UF/DF step, the thawed plasma was fed into the UF/DF membrane cassette and Tris-borate buffer $(\mathrm{pH} \mathrm{8.9)}$ was added to maintain the circulating volume in the system. The UF/DF step was completed once the conductivity of the pooled plasma was below $1000 \mu \mathrm{s} / \mathrm{cm}$.

\section{PRIME process for albumin and immunoglobulin purification}

The pooled plasma after UF/DF was loaded to stream 1 (S1), and Tris-borate buffer was loaded into stream 2 (S2) of the PRIME process. The extraction of albumin using PRIME was completed in approximately $12 \mathrm{hr}$. The product was collected for the analysis of quantity and purity. The albumin-depleted plasma, the intermediate of the PRIME process, was used as the raw material for further purification of IVIG. UFDF was performed using histidine acetate buffer ( $\mathrm{pH}$ 4.5) for the buffer exchange. Post UFDF, samples were loaded into stream 2 (S2) while histidine acetate was loaded into stream 1 (S1) of the PRIME process. The PRIME process took about $12 \mathrm{hr}$ to complete and the product was harvested every $30 \mathrm{~min}$ and collected for the analysis of quantity and purity. 


\section{Sample analysis}

Concentrations of the samples (product yield) were analysed by Bradford assay. Standard calibration curve was made using human albumin (EP) reference standards. Only narrow concentrations of albumin standard were used ( 0 to $2 \mathrm{mg} / \mathrm{ml})$ in order to create an accurate standard curve, which was then used to determine the concentration of the samples. IgG sample concentrations were measured using human IgG (EP) reference standards. iMark Microplate reader (from BioRad) was used to measure the sample absorbances. Sample purity was determined by Size Exclusion (SEC) chromatography (quantitative) and reduced SDS-Page (qualitative). Prominence HPLC (from Schimadzu) and hydrophilic silica gel (as stationary phase) column were used for the sample purity analysis. Reduced SDS-PAGE analysis was used to determine the qualitative purity of the albumin samples and non-reduced SDS-PAGE analysis were used for IVIG samples. Process yield were determined by dividing the total amount of albumin obtained from PRIME process by the total amount of albumin before the PRIME process. The amount of albumin was determined by multiplying the total amount of protein as measured by Bradford Assay and its corresponding purity measured by the SEC-HPLC.

\section{RESULTS AND DISCUSSION}

\section{PRIME process can be used to isolate albumin with purity} and yield of over $90 \%$

In order to purify plasma proteins from source plasma, we designed a process in which albumin, accounting for $50-60 \%$ of plasma proteins [3], is purified first and albumin-depleted plasma is subsequently used for the purification of immunoglobulins (Fig. 2).

The steps for albumin purification include plasma thawing, plasma pooling, post-thaw filtration, ultrafiltration step, PRIME process, chromatography steps, $\mathrm{pH}$ adjustment and heat treatment, ultrafiltration step, bottle filling process, pasteurisation, and final bulk filtration.

We performed five different runs for the purification of albumin and assessed purity of the samples qualitatively using reduced SDS-PAGE (Fig. 3a). SEC HPLC was used to check the quantitative purity of the samples as shown in the chromatogram in Fig. 3b. Our data show that we were able to purify albumin in all five runs with above $90 \%$ purity prior to any polishing steps (e.g., chromatography).

We also tested the yield of the albumin samples from the five runs using both Bradford assay and SEC HPLC (Fig. 3c). Our data show that a consistent yield of over $90 \%$ for albumin for all five runs was obtained.

\section{PRIME process can be used to isolate immunoglobulins} with purity and yield between $\mathbf{8 5 - 9 0 \%}$

Following the isolation of albumin, the albumin-depleted plasma (ADP) was used as the starting material to purify immunoglobulins (IVIG) for two different runs. Results show that we were able to purify IVIG with a process yield of $85-90 \%$ as measured by both Bradford assay and SEC HPLC analysis (Fig. 4a). SEC HPLC data also show a similar result (Fig. 4b).

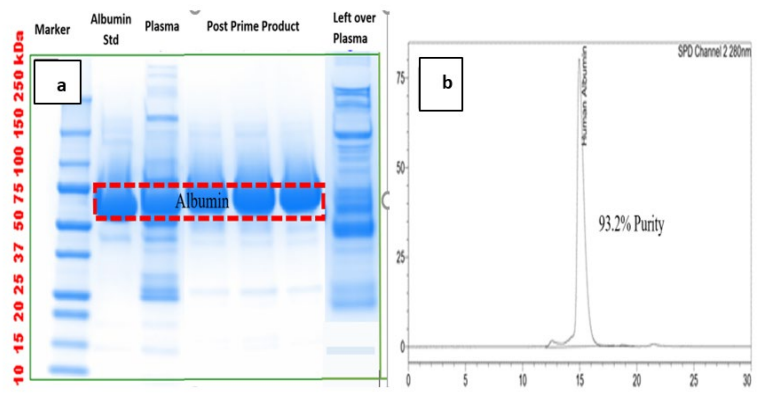

Fig. 3 (a) SDSPAGE image of five runs of albumin purification from source plasma using PRIME technology. Red box indicates albumin; (b) HPLC chromatogram of albumin sample.

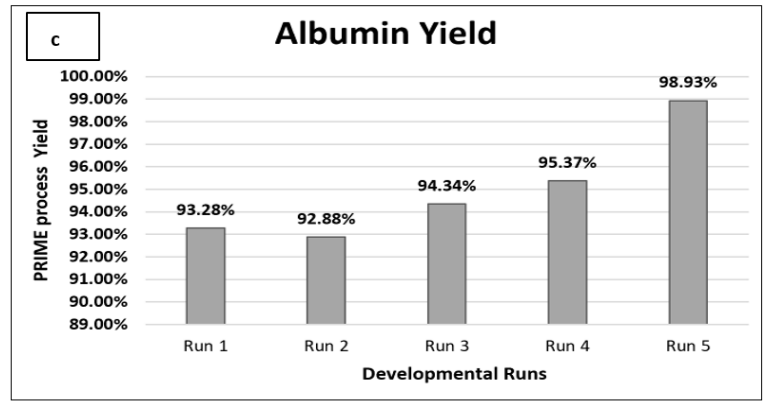

Fig. 3 (c) Percent process yield from five runs of purification of albumin from human plasma using PRIME technology.

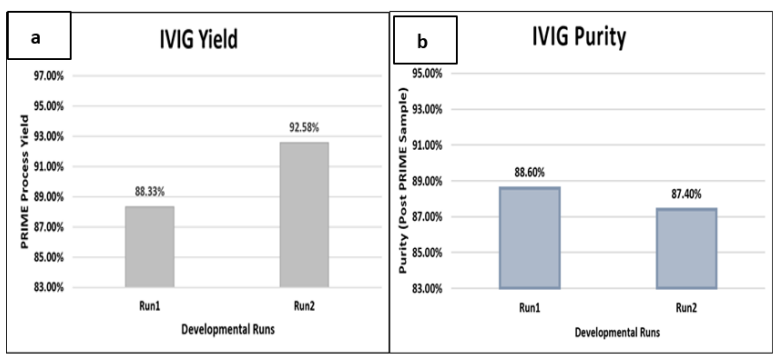

Fig. 4 Results of two runs of IVIG purification from source plasma using PRIME technology. (a) Percent process yield (b) percent process purity.

\section{CONCLUSION}

In this article, we have shown that PRIME technology is capable of purifying albumin and immunoglobulins (IgG) from human plasma at above $80 \%$ and $90 \%$ yield and purity, respectively. It is apparent that PRIME technology has several key benefits that make it an advantageous alternative to Cohn fractionation for the purification of plasma proteins for therapeutic purposes. In addition to albumin and IVIG, we propose that PRIME technology can be used for the purification of clotting factors, specific antibodies, and other plasma proteins.

\section{ACKNOWLEDGEMENT}

This article is supported by PRIME Biologics Pte Ltd, Singapore. All the scientific data, technical knowledge and its intellectual properties and rights are owned by PRIME Biologics Pte. Ltd., Singapore.

We would like to acknowledge Bruce Cohen, Ng Kheng Tee, Wong Lu Er, Chinnusamy Murugan, Lin Jin, Chua Wang Shu, Senthil Kumar, and Edgar Lai for their support throughout this research.

\section{REFERENCES}

[1] Farrugia A. The global need for plasma protein therapies. How much is enough? https://www.pptaglobal.org/images/presentations/7Globaldemand.pdf

[2] Burnouf T. An overview of plasma fractionation. Ann Blood 2018;3: 33

[3] Nicholson JP et al., The role of albumin in critical illness. Br J Anaesth 2000; 85: 599-610 